Thorakal-restriktive Erkrankungen

\section{Bei ineffektiver Heimbeatmung steigt die Sterblichkeit}

Marti S et al. Predictors of mortality in chest wall disease treated with noninvasive home mechanical ventilation. Respir Med. 2010;104:1843-1849

\section{Hintergrund}

Die nicht invasive Heimbeatmung hat bei Patienten mit thorakal-restriktiven Erkrankungen einen hohen Stellenwert. Durch die überwiegend nächtlich durchgeführte Beatmungstherapie ist eine Verbesserung von Symptomen der Hypoventilation, der Blutgase, der Lebensqualität, des Schlafprofils, der Belastbarkeit und der pulmonalen Hämodynamik zu erreichen. Darüber hinaus fanden sich in einigen Untersuchungen Hinweise auf ein verbessertes Langzeitüberleben unter einer nächtlichen Heimbeatmungstherapie.

Nur wenige Studien haben sich mit Prognoseparametern für thorakal-restriktive Erkrankungen auseinandergesetzt. Diese könnten aber hilfreich sein, um die Therapie und das Assessment dieser Patienten zu optimieren.

\section{Methodik}

In die prospektive Studie wurden 110 Patienten mit Posttuberkulosesyndrom oder Kyphoskoliose eingeschlossen, bei denen im Zeitraum von 1996 bis 2006 eine Heimbeatmung begonnen wurde. Die Patienten wurden regelmäßigen Kontrolluntersuchungen zunächst nach einem Monat und dann in drei- bis sechsmonatigen Abständen unterzogen. Alle Patienten wurden, wenn möglich, bis Ende 2007 nachbeobachtet.

\section{Ergebnisse}

$28,1 \%$ der Patienten starben, überwiegend an respiratorischen Erkrankungen. In der univariaten Analyse waren die Komorbiditäten, schlechtere Lungenfunktionswerte sowie ein niedriger Sauerstoffpartialdruck $\left(\mathrm{PaO}_{2}\right)$, aber ein höherer Kohlendioxidpartialdruck $\left(\mathrm{PaCO}_{2}\right)$ nach einem Monat Beatmung mit einem schlechteren Langzeitüberleben assoziiert. In der multivariaten Analyse zeigten sich die Komorbiditäten und der $\mathrm{PaCO}_{2}$ (nach einem Monat) als alleinige unabhängige Prädiktoren. Insbesondere war ein $\mathrm{PaCO}_{2} \geq 50 \mathrm{mmHg}$ mit einem dreifach höheren Mortalitätsrisiko verbunden (Abb. 1).

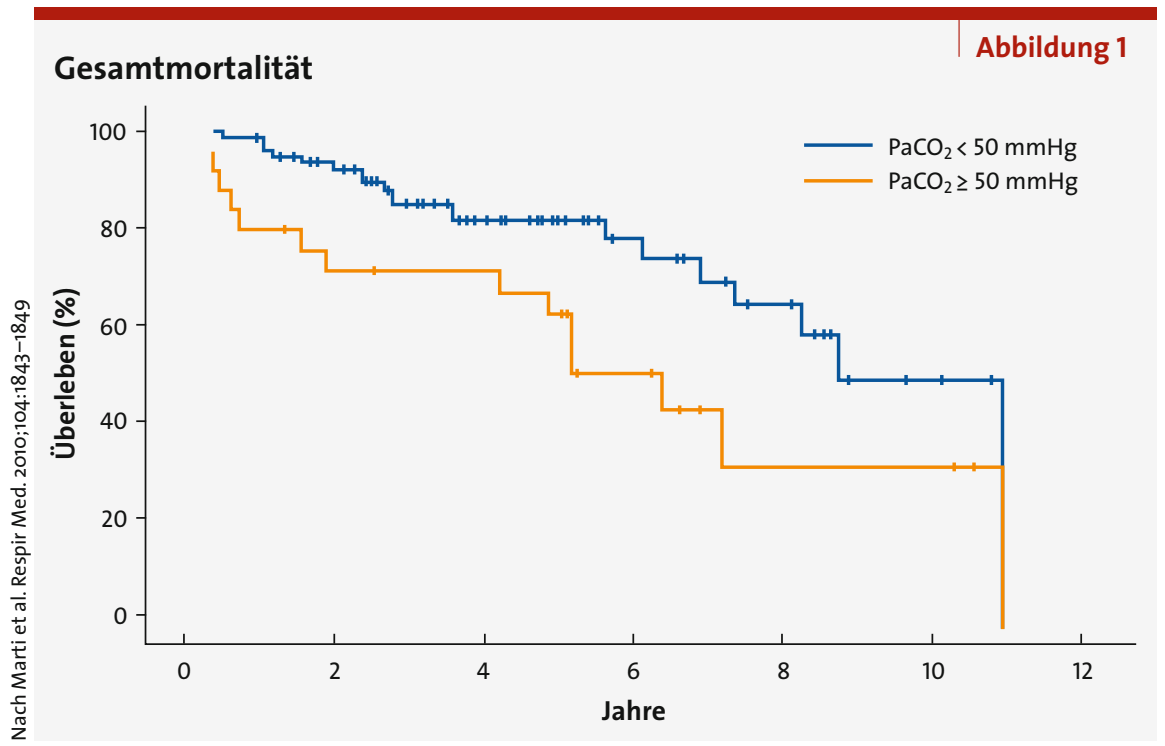

\section{Schlussfolgerung}

Bei Patienten mit thorakal-restriktiven Erkrankungen sind die Komorbiditäten und persistierend hohe $\mathrm{PaCO}_{2}$-Werte $\geq 50 \mathrm{mmHg}$ nach der Einleitung einer Heimbeamtungstherapie unabhängige Prognosefaktoren.

\section{Kommentar}

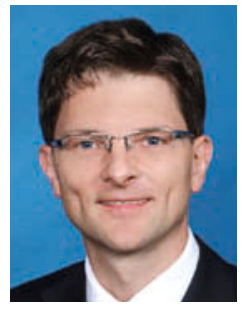

Priv.-Doz. Dr. med. Stephan Budweiser, Rosenheim

Die Indikation und der klinische Nutzen einer nicht invasiven Heimbeatmungstherapie sind bei Patienten mit thorakalrestriktiven Erkrankungen unumstritten. Die kürzlich publizierte S2-Leitline der deutschen Gesellschaft für Pneumologie und Beatmungsmedizin sieht die Indikation für die Heimbeatmung bei diesen Patienten insbesondere dann gegeben, wenn bei Vorliegen entsprechender Symptome der $\mathrm{PaCO}_{2}$ am Tag $>45 \mathrm{mmHg}$ bzw. in der Nacht $>50 \mathrm{mmHg}$ liegt oder ein nächtlicher Anstieg des transkutan gemessenen $\mathrm{CO}_{2}$ um über $10 \mathrm{mmHg}$ dokumentiert werden kann [1]. Darüber hinaus werden bei diesen $\mathrm{Pa}$ tienten nach Einleitung einer Heimbeatmung, unabhängig von der zugrundelie-

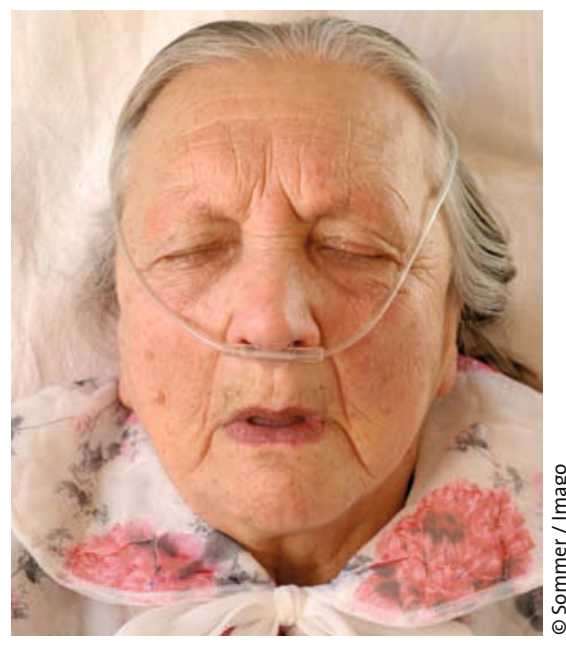

Komorbiditäten und der $\mathrm{PaCO}_{2}$ beeinflussen die Prognose nach Beginn einer Heimbeatmung. 
genden Erkrankung, Kontrolluntersuchungen zunächst nach vier bis acht Wochen und dann ein- bis zweimal jährlich empfohlen. Studien, die dieses Vorgehen wissenschaftlich absichern, fehlen allerdings weitgehend.

In der vorliegenden Arbeit hatten insbesondere Patienten mit einem höheren $\mathrm{PaCO}_{2}$, gemessen einen Monat nach der Einleitung der Heimbeatmung, ein schlechteres Langzeitüberleben. Damit unterstützen diese Daten die Empfehlung der zu- nächst kurzfristigen Beatmungskontrolluntersuchung, um bei entsprechend hohen $\mathrm{CO}_{2}$-Werten die Beatmungseffektivität, beispielsweise durch eine Anpassung der Beatmungsparameter, weiter verbessern zu können. Um tatsächlich die Effektivität der Beatmung überprüfen zu können, sind in der Regel allerdings, wie in der deutschen S2-Leitlinie gefordert, auch nächtliche Blutgasmessungen notwendig. Dabei sollte, wann immer möglich, eine Normoventilation angestrebt werden, die dann, wie die aktuelle Studienlage zeigt, meist von einer guten Therapieadhärenz und Lebensqualität begleitet ist.

\section{Literatur}

1. Windisch $\mathrm{W}$ et al. Non-invasive and invasive mechanical ventilation for treatment of chronic respiratory failure. S2-Guidelines published by the German Medical Association of Pneumology and Ventilatory Support. Pneumologie 2010;64:207-240

\section{Pulmonale Hypertonie}

\section{Statin langfristig ohne Nutzen}

Wilkins MR et al. Simvastatin as a treatment for pulmonary hypertension trial.

Am J Respir Crit Care Med. 2010;181:1106-1113

\section{Hintergrund}

In Tiermodellen hat Simvastatin zu einer Reduktion der pulmonalarteriellen Hypertonie (PAH) und der rechtsventrikulären Hypertrophie geführt $[1,2]$.

\section{Fragestellung}

Diese Studie untersuchte den therapeutischen Wert von Simvastatin bei Patienten mit einer PAH.

\section{Methodik}

In der prospektiven, randomisierten, doppelblinden klinischen Studie erhielten 42 Patienten mit PAH entweder $80 \mathrm{mg} / \mathrm{d}$ Simvastatin oder Placebo zusätzlich zu ihrer Standardtherapie.

\section{Ergebnisse}

In der Simvastatingruppe zeigte sich nach sechs Monaten eine signifikante Reduktion der rechtsventrikulären Masse um 5,2 g, unter Placebo um 3,9 g. Außerdem kam es unter Simvastatin zu einer signifikanten Abnahme der Spiegel des $\mathrm{N}$-terminalen pro-brain-natriuretischen Peptids (NT-proBNP).

In der Erweiterungsstudie über weitere sechs Monate stiegen sowohl die Masse des rechten Ventrikels als auch die NTproBNP-Spiegel bei den 16 Patienten, die von Anfang an Simvastatin erhielten, wieder auf den Ausgangswert. Die Werte bei den 18 Patienten der Placebogruppe, die auf Simvastatin wechselten, blieben stabil.

\section{Schlussfolgerung}

Die Ergebnisse der Studie sprechen gegen einen routinemäßigen Einsatz von Simvastatin in der Therapie der PAH. Simvastatin kann die Progression der Erkrankung zunächst verzögern, aber der langfristige Einfluss ist zu gering.

\section{Kommentar}

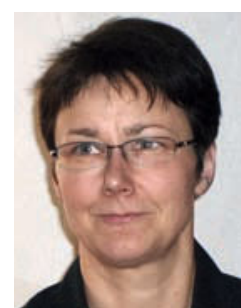

Dr. med. Kathleen Gutjahr, Leipzig

In den letzten Jahren wurden große Fortschritte in der PAH-Therapie erzielt. Die derzeit zugelassenen Medikamente wirken überwiegend vasodilatatorisch und in geringem Maße antiproliferativ. Aufgrund der immer noch schlechten Prognose der Erkrankung ist eine Weiterentwicklung der bestehenden Therapien und die Neuentwicklung therapeutischer Strategien dringend notwendig.

Im Tierexperiment konnte für Simvastatin gezeigt werden, dass sich eine induzierte PAH durch die Induktion der Apoptose der glatten Muskelzellen der Neointima bessert.

Basierend auf diesem Ergebnis haben Kao et al. 2005 eine Fallserie veröffentlicht [2]. 16 Patienten mit einer PAH der WHOKlassen 1-4 wurden auf 20-80 mg/d Simvastatin eingestellt. Simvastatin erwies sich als sicher, und es wurde die Hoffnung geweckt, dass Simvastatin einen positiven Effekt auf den Verlauf der PAH hat.

Der Benefit von Simvastatin bei der PAH bleibt laut den Ergebnissen dieser Studie nicht erhalten. Allerdings wurden die inzwischen üblichen Ziele der PAH-Therapie, die Erhöhung der Belastungstoleranz, gemessen an der 6-Minuten-Gehstrecke, hier nicht getestet, sodass diesbezüglich weitere Untersuchungen notwendig wären. 